\title{
EFFICACITÉ D'UN EXUTOIRE DE DÉVALAISON POUR SMOLTS DE SAUMON ATLANTIQUE (SALMO SALAR L.) ET COMPORTEMENT DES POISSONS AU NIVEAU DE L'AMÉNAGEMENT HYDROÉLECTRIQUE DE CAMON SUR LA GARONNE.
}

\author{
O. CROZE, M. CHANSEAU et M. LARINIER.
}

CSP - CEMAGREF - GHAAPPE, Institut de Mécanique des Fluides, Avenue du Professeur Camille Soula, 31400 Toulouse, France.

Reçu le 19 mai 1999

Accepté le 03 juin 1999
Received 19 May, 1999

Accepted 03 June, 1999

\section{RÉSUMÉ}

Trois expérimentations ont été conduites de 1996 à 1998 au niveau de la prise d'eau de l'usine hydroélectrique de Camon, sur la Garonne, de manière à tester l'efficacité d'un exutoire de surface destiné aux smolts de saumon atlantique. Ce dispositif de dévalaison est situé en rive gauche, au niveau du plan des grilles. Son efficacité a été évaluée par la méthode de marquage-recapture et le comportement des smolts dans le canal d'amenée a été suivi grâce à la technique de radio-pistage.

L'efficacité initiale du dispositif est apparue faible en 1996 (moyenne de $34 \%$ ). Le suivi par radio-pistage a révélé que l'emplacement de l'exutoire n'était pas responsable de sa faible efficacité, les poissons stationnant préférentiellement à proximité. Cependant, la présence d'une ascendance masque son entrée. Suite à la mise en place d'une grille de tranquillisation et de platelages horizontaux au niveau de l'entrée de l'exutoire, cette efficacité a été portée à une valeur moyenne de $73 \%$. De bonnes conditions hydrauliques générales dans le canal d'amenée associées à une hydrodynamique locale favorable aux environs immédiats de l'entrée de l'exutoire s'avèrent indispensables à l'obtention d'une efficacité satisfaisante du dispositif de dévalaison.

L'utilisation d'un éclairage nocturne intermittent a non seulement eu un effet sur le comportement des smolts dans le canal d'amenée en maintenant ces derniers dans les zones directement éclairées mais aussi sur les rythmes d'entrée des poissons dans l'exutoire, les passages apparaissant principalement en début d'extinction.

La capture de 7715 salmonidés sauvages a permis d'étudier les rythmes de dévalaison au niveau d'un obstacle. Les pics journaliers de passage semblent liés principalement à de forts débits et/ou à une augmentation de la température de l'eau. L'activité de dévalaison au niveau d'un obstacle apparaît en outre essentiellement nocturne.

Mots-clés : dévalaison, exutoire de surface, smolt, saumon atlantique, usine hydroélectrique, marquage-recapture, radio-pistage, comportement, lumière, hydrodynamique. 


\section{EFFICIENCY OF A DOWNSTREAM BYPASS FOR ATLANTIC SALMON (SALMO SALAR L.) SMOLTS AND FISH BEHAVIOUR AT THE CAMON HYDROELECTRIC POWERHOUSE WATER INTAKE ON THE GARONNE RIVER.}

\section{ABSTRACT}

Three experiments were conducted from 1996 to 1998 at the Camon hydroelectric powerhouse water intake, on the Garonne River, to test the efficiency of a surface downstream bypass for Atlantic salmon smolts. This bypass was built into the trashrack itself at its left edge. The efficiency of the device was evaluated using the mark-recapture method. Smolt behaviour in the intake canal was studied using radiotelemetry technique.

In 1996, the bypass efficiency was low (34\%). Radio-tracking showed that the bypass location was not responsible for its low efficiency, fish being listened most of the time in the vicinity of the bypass. Nevertheless, an unstable upwelling hided the device entrance. After installing submerged horizontal screen and plates upstream bypass entrance gate, the average efficiency increased to $73 \%$. Good hydraulic conditions in the intake canal and good local hydrodynamic in the vicinity of the bypass entrance are essential to obtain a satisfactory downstream bypass efficiency.

Intermittent nocturnal lighting has an effect on smolt behaviour in the intake canal by maintaining fish in directly lighted areas and on the rhythm of fish entry in the bypass, more fish being captured during the first part of the lighting off period.

The catching of 7,715 wild salmonids has permitted to study downstream migration rhythms at dam. Daily downstream migration peaks seems to be linked with high water discharge and/or an increase of water temperature. Moreover, downstream migration activity at a dam appears to be mainly nocturnal.

Key-words : downstream migration, surface bypass, smolt, Atlantic salmon, hydroelectric powerhouse, mark-recapture, radio-tracking, behaviour, light, hydrodynamics.

\section{INTRODUCTION}

Les expérimentations conduites de 1996 à 1998 à l'usine hydroélectrique de Camon, sur la Garonne, s'inscrivent dans un programme à long terme visant à définir les critères de conception et les limites de fonctionnement des exutoires de surface au niveau des prises d'eau des centrales hydroélectriques. Elles étaient destinées à évaluer l'efficacité d'un dispositif de dévalaison installé à la prise d'eau de l'usine, cette dernière étant beaucoup plus large que celles qui avaient fait l'objet de tests les années précédentes au niveau des usines d'Halsou sur la Nive (LARINIER et BOYER-BERNARD, 1991a), de Poutès sur l'Allier (LARINIER et BOYER-BERNARD, 1991b), de Soeix (TRAVADE et al., 1996) et de Bedous (CHANSEAU, LARINIER et TRAVADE, 1999) sur le Gave d'Aspe.

\section{MATÉRIEL ET MÉTHODES}

\section{Site d'étude}

L'aménagement hydroélectrique de Camon est situé dans le sud-ouest de la France, sur la Garonne, à $90 \mathrm{~km}$ de la source et $115 \mathrm{~km}$ en amont de Toulouse (Figure 1). Au niveau de l'aménagement, le bassin versant naturel est voisin de $2100 \mathrm{~km}^{2}$. Le régime 
des eaux, de type pluvio-nival, se caractérise par de forts débits en hiver et surtout au printemps ainsi que par de basses eaux d'août à octobre. Le module interannuel au niveau du site est voisin de $52 \mathrm{~m} 3 / \mathrm{s}$. Les débits moyens mensuels pour les mois de dévalaison sont de $50 \mathrm{~m}^{3} / \mathrm{s}$ en mars, $73 \mathrm{~m}^{3} / \mathrm{s}$ en avril et $97 \mathrm{~m}^{3} / \mathrm{s}$ en mai.

L'aménagement de Camon comprend un barrage mobile (Rodère) de $66 \mathrm{~m}$ de longueur et d'une hauteur de $6 \mathrm{~m}$ qui court-circuite la Garonne sur $7 \mathrm{~km}$ (Figure 1) pour alimenter, par un canal de $3,4 \mathrm{~km}$ de longueur, une centrale hydroélectrique (Figure 2A). Cette centrale est équipée de trois turbines Francis (puissance nominale : $5200 \mathrm{~kW}$ par turbine, chute nette : $21,45 \mathrm{~m}$ ). Le débit maximum turbinable est de $85 \mathrm{~m}^{3} / \mathrm{s}$. Une seconde usine (Valentine) est située sur la même dérivation, à environ $3 \mathrm{~km}$ en aval de l'usine de Camon.
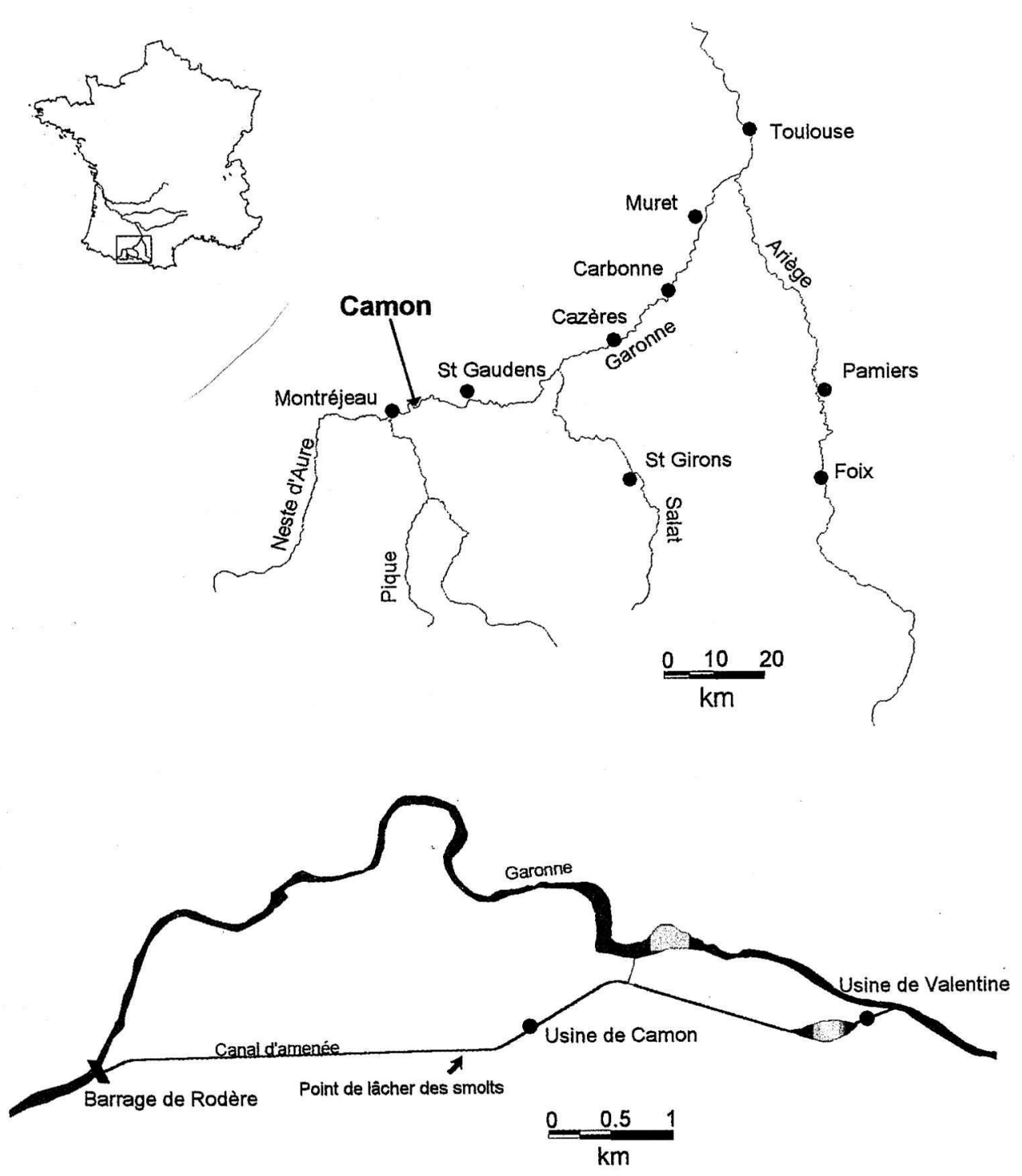

\section{Figure 1}

Situation géographique et vue générale de l'aménagement hydroélectrique de Camon.

Figure 1

Geographical situation and general view of Camon hydroelectric plant. 

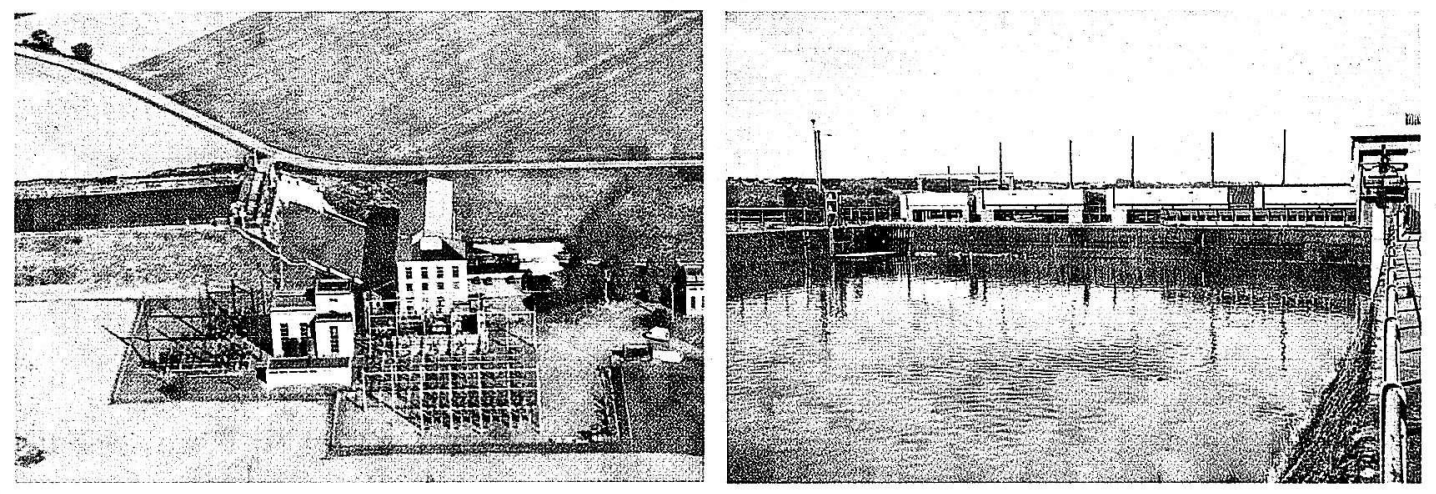

A
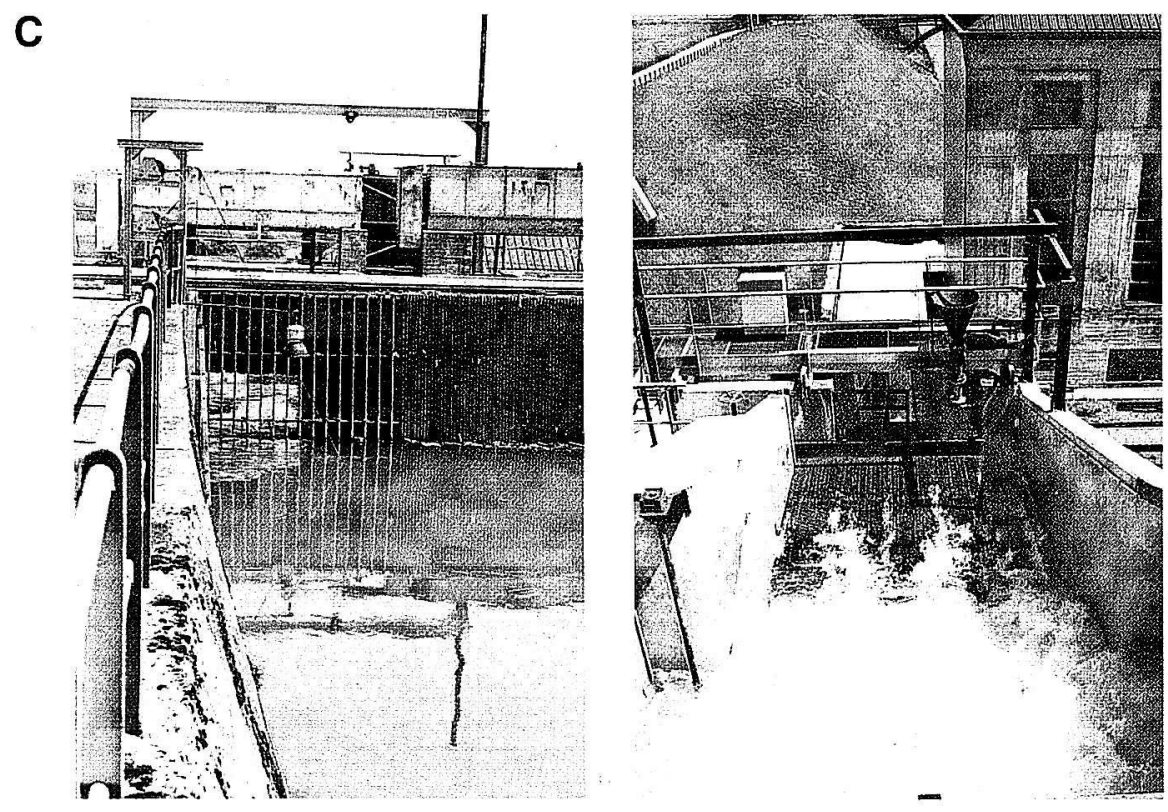

D

Figure 2A

Vue aérienne de l'usine hydroélectrique de Camon.

Figure 2B

Vue amont de la prise d'eau de l'usine de Camon.

Figure 2C

Vue de l'entrée de l'exutoire de dévalaison.

Figure 2D

Vue de l'exutoire avec le bassin de dissipation, la grille de filtration et la goulotte de récupération des poissons.

Figure 2A

Aerial view of Camon hydroelectric powerhouse.

Figure 2B

Upstream view of Camon water intake.

Figure 2C

View of the downstream bypass entrance.

Figure 2D

View of bypass with the stilling pool, the filtering screen and the fish trough. 

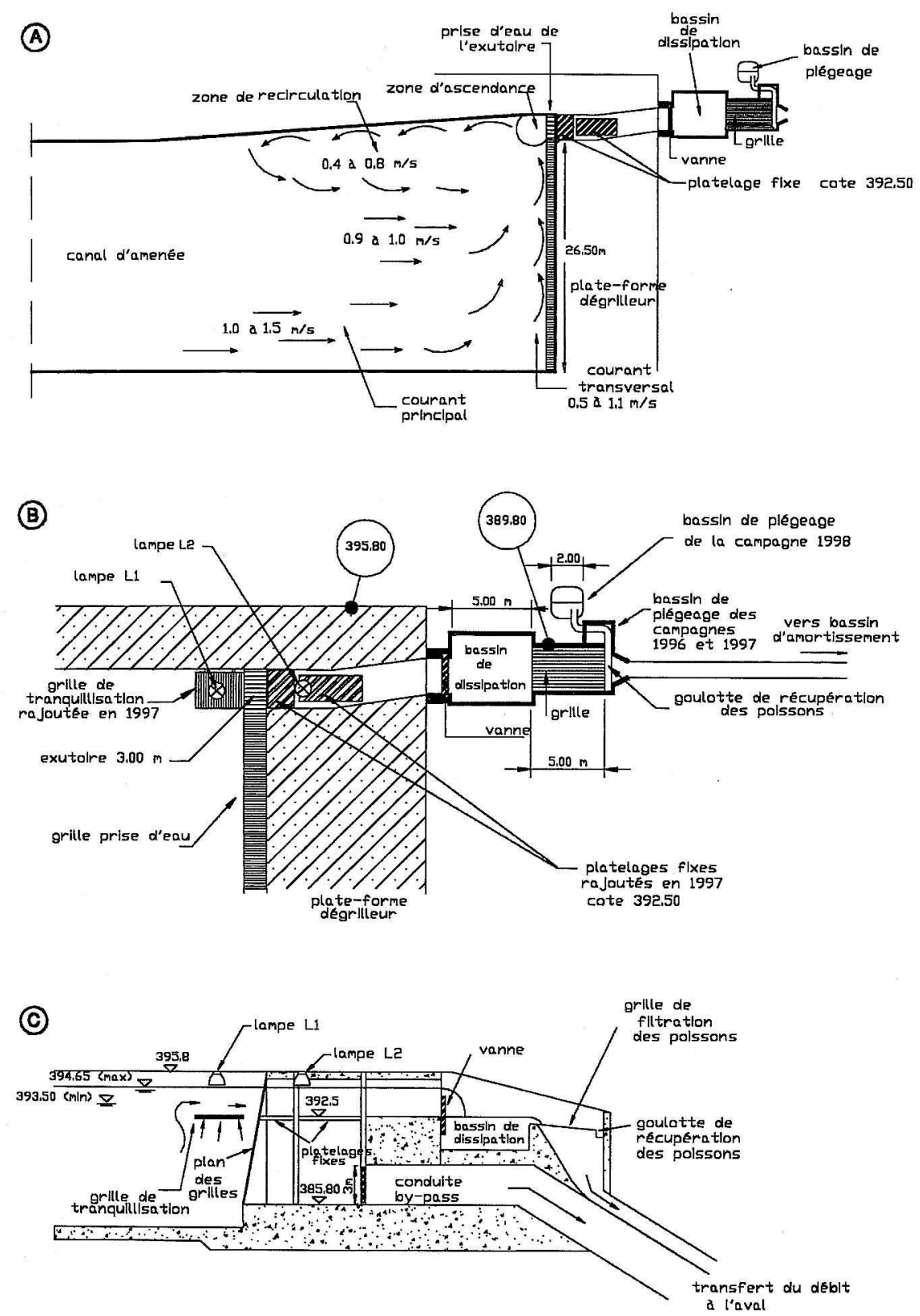

\section{Figure 3A}

Vue générale de la prise d'eau de l'usine hydroélectrique de Camon et hydrodynamique du canal d'amenée.

\section{Figure 3B}

Vue en plan de l'exutoire de dévalaison et du dispositif de piégeage.

\section{Figure $3 \mathrm{C}$}

Vue en coupe de l'exutoire de dévalaison.

\section{Figure 3A}

General view of Camon hydroelectric power intake and velocity patterns in the forebay.

\section{Figure 3B}

Plan view of the downstream migration bypass and trapping facilities.

\section{Figure $3 \mathrm{C}$}

Section view of the downstream migration bypass. 
Le canal d'amenée a une largeur voisine de $20 \mathrm{~m}$, la profondeur d'eau pour la cote de retenue normale étant d'environ $5,5 \mathrm{~m}$. La largeur de la prise d'eau à l'usine est de $29,5 \mathrm{~m}$. Les grilles, d'une hauteur totale de $8,3 \mathrm{~m}$, sont constituées de barreaux rectangulaires présentant un espacement de $4 \mathrm{~cm}$. En rive gauche, sur une largeur de $3 \mathrm{~m}$, la prise d'eau alimente une conduite by-pass de $3 \mathrm{~m}$ de diamètre dont la partie supérieure se trouve à $3 \mathrm{~m}$ sous la cote de retenue normale et qui est équipée d'une vanne plate en tête. Sur cette partie de la prise, l'espacement des barreaux est de $17 \mathrm{~cm}$ (Figure 2B).

Le dispositif de dévalaison a été installé en surface au-dessus de cette conduite by-pass (Figures $2 \mathrm{C}$ et $3 \mathrm{~A}$ ). II se compose d'une vanne plate de $2,3 \mathrm{~m}$ de largeur, alimentant un bassin de dissipation (Figures 2D, 3B et $3 C$ ). Ce bassin communique par l'intermédiaire d'un déversoir avec un canal de $1 \mathrm{~m}$ de largeur et de $38 \mathrm{~m}$ de longueur qui restitue le débit de l'exutoire dans un bassin d'amortissement en communication avec le canal de fuite.

\section{Hydrodynamique en amont de la prise d'eau de l'usine}

L'alimentation de la prise d'eau se fait préférentiellement par la rive droite avec des vitesses d'écoulement maximales principalement localisées sur une bande d'une douzaine de mètres de largeur à partir de cette rive. Les valeurs de vitesse de surface mesurées à l'aide de flotteurs sont comprises entre $1 \mathrm{~m} / \mathrm{s}$ et $1,5 \mathrm{~m} / \mathrm{s}$ et peuvent dépasser ponctuellement $2 \mathrm{~m} / \mathrm{s}$ lorsque le débit dérivé est proche du maximum turbinable. II apparaît au droit des grilles un courant transversal marqué, dirigé vers la rive gauche et dont les vitesses peuvent dépasser $1 \mathrm{~m} / \mathrm{s}$. Ce courant engendre, au voisinage de la prise d'eau de l'exutoire, une zone d'ascendance masquant l'entrée du dispositif de dévalaison (Figure 3A).

Une grille de tranquillisation horizontale de $2,5 \mathrm{~m} \times 3 \mathrm{~m}$ ainsi que deux platelages ont été installés au niveau de l'entrée de l'exutoire en 1997 (Figures 3B et 3C). Ces aménagements avaient pour but de réduire les ascendances en amont immédiat du dispositif de dévalaison et d'augmenter les composantes horizontales des vitesses au niveau de sa prise d'eau de manière à étendre sa zone d'influence.

\section{Périodes d'étude}

Bien qu'initialement prévue pour le début du mois d'avril, l'expérimentation menée en 1996 n'a pu commencer que le 9 mai en raison du retard des travaux relatifs à la construction de l'exutoire. Cette première campagne s'est prolongée jusqu'au 23 mai. La campagne 1997, débutée le 10 avril, s'est achevée le 26 mai, ce qui correspond à 47 jours d'expérimentation. Au cours de la campagne, le piégeage a dû être interrompu près de 8 jours consécutifs (du 29 avril au 7 mai), le canal restituant le débit de l'exutoire à l'aval de l'usine ayant été endommagé. Le suivi de l'exutoire de dévalaison a donc été effectué pendant 39 jours. La campagne 1998 s'est déroulée du 6 avril au 20 mai, soit pendani 45 jours.

\section{Paramètres du milieu}

La température et le niveau d'eau en amont des grilles de la prise d'eau ont fait l'objet d'enregistrements en continu pendant les expérimentations au moyen de centrales d'acquisition portables autonomes. 


\section{Suivi de l'activité de dévalaison}

Un dispositif de piégeage a été intégré à l'exutoire de dévalaison lors de sa conception. Il est constitué d'une grille inclinée (pente $12 \%$ ) qui filtre le débit entrant dans l'exutoire et permet de guider les poissons vers un bassin de piégeage (Figure 3B).

Pendant toute la durée des 3 campagnes, le piège a été relevé 3 fois par jour les jours de lâcher (avant le lâcher, deux heures après le lâcher, le matin suivant le lâcher). Les jours sans lâcher, 2 piégeages quotidiens ont été assurés. A chaque relevé de piège, les poissons ont été identifiés (espèce, relevé de marque) et mesurés (longueur totale).

Une surveillance vidéo nocturne des passages de poissons dans l'exutoire a été assurée en 1996 et 1997 grâce à une caméra installée à l'aplomb de l'exutoire de dévalaison, dans l'axe de la grille de filtration. Cette caméra était reliée à un magnétoscope à temps échelonné (Panasonic AG-6720A-E). En 1998, cette même caméra a été placée au-dessus de la goulotte de récupération des poissons et a fonctionné de jour comme de nuit.

\section{Contrôle de l'efficacité de l'exutoire de dévalaison}

L'efficacité de l'exutoire a été évaluée grâce à des opérations de marquage-recapture de smolts de saumon atlantique. Les poissons déversés provenaient d'une pisciculture, la population dévalante issue d'alevinages ou d'une reproduction naturelle de géniteurs transportés sur la partie amont de la Garonne étant quasi inexistante en 1996 et 1997 ou peu importante et difficilement capturable en amont de Camon en 1998.

Les protocoles de marquage et de lâcher sont semblables à ceux utilisés par CHANSEAU, LARINIER et TRAVADE (1999). Les lâchers ont été effectués par lots d'environ 200 individus en 1996 et d'une centaine d'individus en 1997 et 1998 . Onze lots ont pu être constitués lors de la première campagne, 18 lots lors de la seconde et 14 lots lors de la troisième. Au total, 4825 smolts ont été lâchés dont 2025 en 1996, 1528 en 1997 et 1272 en 1998.

Les poissons ont été déversés en rive droite du canal d'amenée, $550 \mathrm{~m}$ en amont de la prise d'eau de l'usine, une fois la nuit tombée. Seuls 3 lots ont été lâchés en journée lors de la première campagne.

\section{Comportement des smolts}

Le comportement de 11 smolts marqués à l'aide d'un émetteur a été étudié en 1996 grâce à la technique de radio-pistage. Le matériel utilisé est identique à celui employé par CHANSEAU, LARINIER et TRAVADE (1999). Quinze zones de réception ont été individualisées par des antennes, de la prise d'eau de l'usine jusqu'à $50 \mathrm{~m}$ en amont (Figure 4) : les déplacements des poissons radiomarqués ont été enregistrés en continu sur les 50 derniers mètres du canal d'amenée. Ces poissons, issus de pisciculture, ont été lâchés au sein des lots destinés aux opérations de marquage-recapture à raison d'un seul poisson radiomarqué par lot.

En plus de la technique de radio-pistage, l'utilisation d'un échosondeur (Lowrance $\mathrm{X}$-16) a permis de mettre en évidence la présence éventuelle de poissons au voisinage de l'entrée du dispositif de dévalaison. Ce matériel, mis en fonctionnement pendant une période d'une à deux heures après les lâchers, n'a été employé qu'au cours de la première campagne. 


\section{Débits de l'exutoire}

La vanne plate alimentant en eau l'exutoire de dévalaison est asservie au niveau amont et permet de maintenir un débit entrant constant. Ce débit peut prendre trois valeurs définies, voisines de 2,3 et $4 \mathrm{~m}^{3} / \mathrm{s}$.

Lors des études 1996 et 1997, le débit dans l'exutoire a été fixé à 2 m³/s et porté à $3 \mathrm{~m} 3 / \mathrm{s}$, pour la plupart des lots, pendant la période séparant le lâcher du premier piégeage. En 1998, un débit constant de $3 \mathrm{~m} 3 / \mathrm{s}$ a pu être délivré à l'exutoire de Camon pendant la totalité de la campagne.

\section{Influence de la lumière}

De nombreuses expérimentations ont mis en évidence l'effet attractif d'un éclairage nocturne par des lampes à vapeur de mercure de faible puissance sur les poissons et notamment sur les smolts en dévalaison (TAFT, 1988 ; LARINIER et BOYER-BERNARD, 1991a ; EPRI, 1994).

Deux lampes à vapeur de mercure (puissance $80 \mathrm{~W}$ ) ont été installées sur le site de Camon, la première (L1) à $2 \mathrm{~m}$ en amont de la grille de protection de la prise d'eau de l'exutoire et à $1,5 \mathrm{~m}$ de la rive gauche, la seconde lampe (L2) à 2,2 $\mathrm{m}$ en aval de cette grille (Figures $3 \mathrm{~B}$ et $3 \mathrm{C}$ ).

Au cours des deux premières campagnes, une seule lampe à vapeur de mercure a été utilisée, L1 en 1996 et L2 en 1997. Ces lampes ont répété des cycles d'une durée totale d'un quart d'heure comprenant une période d'éclairage (10 minutes) suivie d'une période d'extinction (5 minutes). En 1998, un nouveau cycle lumineux d'une durée totale de 15 minutes et faisant intervenir les deux lampes a été testé. Pendant les 8 premières minutes du cycle, L1 et L2 étaient allumées simultanément. Au cours des 5 minutes suivantes, seule la lampe éclairant l'aval de la grille de l'exutoire fonctionnait. Les lampes étaient toutes deux éteintes pendant les deux dernières minutes. Ces conditions d'éclairage étaient maintenues toute la nuit.

\section{RÉSULTATS}

\section{Evaluation de l'efficacité de l'exutoire et comportement des smolts en 1996}

\section{Efficacité de l'exutoire de dévalaison}

Sur les 2025 smolts lâchés, 571 ont été récupérés dans le piège, ce qui correspond à une efficacité moyenne de l'exutoire de dévalaison de $28,2 \%$. Selon les lots, le pourcentage de recapture a varié de $1 \%$ à $76 \%$ (Tableau I). Plusieurs incidents affectant le fonctionnement de la vanne de l'exutoire ont cependant perturbé 2 des 11 lâchers. L'efficacité du dispositif de dévalaison, calculée en retirant ces deux lots, a varié de $7 \%$ à $76 \%$ selon les essais et atteint une valeur moyenne de 33,8 \% (550 smolts piégés sur 1625 lâchés).

Le débit turbiné à Camon a varié de $48 \mathrm{~m} 3 / \mathrm{s}$ à $85 \mathrm{~m} 3 / \mathrm{s}$ au cours de la campagne 1996. Le débit de l'exutoire étant de $3 \mathrm{~m}^{3} / \mathrm{s}$ pendant les 2 heures suivant le lâcher de 7 des 11 lots et limité à $2 \mathrm{~m}^{3} / \mathrm{s}$ le reste du temps, il représente entre $2 \%$ et $4 \%$ du débit turbiné.

\section{Comportement des smolts}

Grâce à la technique de radio-pistage, les déplacements de 11 smolts ont pu être suivis depuis leur lâcher jusqu'à leur passage en aval de l'usine. 
Le suivi télémétrique a permis de mettre en évidence un trajet type des smolts marqués d'un radio-émetteur (Figure 4). Du point de lâcher jusqu'à la prise d'eau, les poissons dévalent dans la veine de courant principal. Les poissons arrivaient ainsi préférentiellement par la rive droite, le courant transversal présent en amont des grilles de la prise d'eau de l'usine les guidant alors vers le milieu du canal (zone 5), puis vers la rive gauche (zone 3 ). Les smolts demeuraient alors à proximité de l'exutoire (zones 2 et 3 ). Ils quittaient ce lieu de stationnement en suivant le contre-courant localisé en rive gauche et se dirigeaient vers l'amont à des distances comprises entre $10 \mathrm{~m}$ et $40 \mathrm{~m}$ des grilles de la prise d'eau. Ils se déplaçaient ensuite vers le milieu du canal et dévalaient de nouveau pour se présenter au niveau des grilles plutôt par la zone 5 . Un tel parcours a pu être réalisé de nombreuses fois par le même individu.

Les poissons n'ont pas fréquenté avec la même intensité les différentes zones de réception (ANOVA, $F=21,43 ; p<0,001$ ). Les zones où les smolts stationnaient préférentiellement étaient celles situées à proximité de l'exutoire (Figure 4); ils passaient en effet respectivement $52 \%$ et $17 \%$ de leur temps de présence sur site au niveau des zones 2 et 3 . Les zones situées en rive droite étaient les moins fréquentées. Un seul smolt a pénétré dans la zone 9 et uniquement 2 poissons ont été localisés au niveau de la zone 7.

Une fois les grilles atteintes, les smolts sont restés de 4 min à 3 h 17 min dans le canal d'amenée (médiane de $34 \mathrm{~min}$ ) avant de dévaler par la prise d'eau de l'usine ou d'entrer dans l'exutoire.

II convient de noter que tous les smolts radiomarqués ont pénétré à plusieurs reprises dans la zone de réception située en amont immédiat de l'entrée de l'exutoire (zone 2), le nombre d'incursions dans cette zone variant de 3 à 44 selon les individus. Un échosondeur installé à ce niveau a montré que les smolts évoluaient de préférence dans les $2,2 \mathrm{~m}$ supérieurs de la colonne d'eau.

\section{Tableau 1}

Récapitulatif des opérations de marquage-recapture et résultats d'efficacité de l'exutoire pour la campagne 1996.

\section{Table I}

Summary of mark-recapture operations and results of bypass efficiency for 1996 study.

CAMPAGNE 1996

\begin{tabular}{|c|c|c|c|c|c|}
\hline Date et heure de lâcher & $\begin{array}{l}\text { Nombre de } \\
\text { smolts lâchés }\end{array}$ & $\begin{array}{l}\text { Nornbre de } \\
\text { smolts piégés }\end{array}$ & $\begin{array}{l}\text { Pourcentage de } \\
\text { smolts piégés }\end{array}$ & $\begin{array}{c}\text { Pannes de la } \\
\text { vanne d'entrée }\end{array}$ & $\begin{array}{l}\text { Débit de } \\
\text { l'exutoire }\end{array}$ \\
\hline 09/05/96 à 23:15 & 200 & 13 & 7 & - & $2 \mathrm{~m}^{3} / \mathrm{s}$ \\
\hline $10 / 05 / 96$ à $18: 57$ & 200 & 19 & 10 & oui & $2 \mathrm{~m}^{3} / \mathrm{s}$ \\
\hline $11 / 05 / 96$ à $16: 58$ & 200 & 2 & 1 & ouj & $2 \mathrm{~m}^{3} / \mathrm{s}$ \\
\hline $13 / 05 / 96$ à $23: 19$ & 192 & 45 & 23 & - & $2 \mathrm{~m}^{3} / \mathrm{s}$ \\
\hline 14/05/96 à 23:39 & 193 & 98 & 51 & - & 3 puis $2 \mathrm{~m}^{3} / \mathrm{s}$ \\
\hline $15 / 05 / 96$ à $18: 49$ & 198 & 64 & 32 & - & 3 puis $2 \mathrm{~m}^{3} / \mathrm{s}$ \\
\hline $16 / 05 / 96$ à $23: 31$ & 195 & 148 & 76 & - & 3 puis $2 \mathrm{~m}^{3} / \mathrm{s}$ \\
\hline $17 / 05 / 96$ à 23:14 & 199 & 57 & 29 & - & 3 puis $2 \mathrm{~m}^{3} / \mathrm{s}$ \\
\hline 20/05/96 à 23:25 & 201 & 38 & 19 & - & 3 puis $2 \mathrm{~m}^{3} / \mathrm{s}$ \\
\hline 21/05/96 à 23:36 & 201 & 63 & 31 & - & 3 puis $2 \mathrm{~m}^{3} / \mathrm{s}$ \\
\hline 23/05/96 à 00:16 & 46 & 24 & 52 & - & 3 puis $2 \mathrm{~m}^{3} / \mathrm{s}$ \\
\hline Total (tous lots) & 2025 & 571 & 28,2 & & \\
\hline Total (sans pannes de vanne) & 1625 & 550 & 33,8 & & \\
\hline
\end{tabular}


Seuls 3 des 11 poissons radiomarqués ont dévalé par l'exutoire. Quatre sont passés à travers les grilles de protection de la prise d'eau des turbines par la zone 3 , soit à moins de $4 \mathrm{~m}$ de l'entrée de l'exutoire, 3 ont franchi ces grilles au niveau de la zone 4 et 1 probablement par le milieu du canal.

\section{Tableau II}

Récapitulatif des opérations de marquage-recapture et résultats d'efficacité de l'exutoire pour les campagnes 1997 et 1998.

\section{Table II}

\section{Summary of mark-recapture operations and results of bypass efficiency for 1997 and 1998 studies.}

\section{CAMPAGNE 1997}

\begin{tabular}{|c|c|c|c|c|c|}
\hline Date et heure de lâcher & $\begin{array}{l}\text { Nombre de } \\
\text { smolts lâchés }\end{array}$ & $\begin{array}{c}\text { Nombre de } \\
\text { smolts piégés }\end{array}$ & $\begin{array}{l}\text { Pourcentage de } \\
\text { smolts piégés }\end{array}$ & $\begin{array}{c}\text { Pannes de la } \\
\text { vanne d'entrée }\end{array}$ & $\begin{array}{l}\text { Débit de } \\
\text { l'exutoire }\end{array}$ \\
\hline $10 / 04 / 97$ à $21: 48$ & 100 & 70 & 70 & - & $2 \mathrm{~m}^{3} / \mathrm{s}$ \\
\hline $14 / 04 / 97$ à $22: 30$ & 100 & 71 & 71 & - & 3 puis $2 \mathrm{~m}^{3} / \mathrm{s}$ \\
\hline $15 / 04 / 97$ à $22: 15$ & 100 & 69 & 69 & - & 3 puis $2 \mathrm{~m}^{3} / \mathrm{s}$ \\
\hline $18 / 04 / 97$ à $23: 00$ & 100 & 72 & 72 & - & 3 puis $2 \mathrm{~m}^{3} / \mathrm{s}$ \\
\hline $21 / 04 / 97$ à $22: 44$ & 100 & 71 & 71 & - & 3 puis $2 \mathrm{~m}^{3} / \mathrm{s}$ \\
\hline 22/04/97 à 23:00 & 100 & 61 & 61 & - & 3 puis $2 \mathrm{~m}^{3} / \mathrm{s}$ \\
\hline $24 / 04 / 97$ à $22: 45$ & 100 & 69 & 69 & - & 3 puis $2 \mathrm{~m}^{3} / \mathrm{s}$ \\
\hline $07 / 05 / 97$ à $22: 45$ & 100 & 74 & 74 & - & 3 puis $2 \mathrm{~m}^{3} / \mathrm{s}$ \\
\hline 08/05/97 à 23:44 & 100 & 83 & 83 & - & 3 puis $2 \mathrm{~m}^{3} / \mathrm{s}$ \\
\hline $12 / 05 / 97$ à $22: 13$ & 100 & 72 & 72 & - & 3 puis $2 \mathrm{~m}^{3} / \mathrm{s}$ \\
\hline $13 / 05 / 97$ à $22: 55$ & 100 & 84 & 84 & - & 3 puis $2 \mathrm{~m}^{3} / \mathrm{s}$ \\
\hline $14 / 05 / 97$ à $22: 54$ & 98 & 77 & 79 & - & 3 puis $2 \mathrm{~m}^{3} / \mathrm{s}$ \\
\hline 15/05/97 à 23:13 & 99 & 77 & 78 & - & 3 puis $2 \mathrm{~m}^{3} / \mathrm{s}$ \\
\hline 20/05/97 à 23:27 & 100 & 77 & 77 & - & 3 puis $2 \mathrm{~m}^{3} / \mathrm{s}$ \\
\hline $21 / 05 / 97$ à $23: 58$ & 96 & 72 & 75 & - & 3 puis $2 \mathrm{~m}^{3} / \mathrm{s}$ \\
\hline $22 / 05 / 97$ à $23: 59$ & 35 & 20 & 57 & - & 3 puis $2 \mathrm{~m}^{3} / \mathrm{s}$ \\
\hline Total (tous lots) & 1528 & 1119 & 73,2 & & \\
\hline
\end{tabular}

\section{CAMPAGNE 1998}

\begin{tabular}{|c|c|c|c|c|c|}
\hline Date et heure de lâcher & $\begin{array}{l}\text { Nombre de } \\
\text { smolts lâchés }\end{array}$ & $\begin{array}{c}\text { Nombre de } \\
\text { smolts piégés }\end{array}$ & $\begin{array}{l}\text { Pourcentage de } \\
\text { smolts piégés }\end{array}$ & $\begin{array}{c}\text { Pannes de la } \\
\text { vanne d'entrée }\end{array}$ & $\begin{array}{l}\text { Débit de } \\
\text { l'exutoire }\end{array}$ \\
\hline 07/04/98 à 00:06 & 75 & 54 & 72 & oui & $3 \mathrm{~m}^{3} / \mathrm{s}$ \\
\hline $08 / 04 / 98$ à $22: 20$ & 100 & 68 & 68 & oui & $3 \mathrm{~m}^{3} / \mathrm{s}$ \\
\hline $09 / 04 / 98$ à $22: 56$ & 100 & 68 & 68 & oui & $3 \mathrm{~m}^{3} / \mathrm{s}$ \\
\hline $14 / 04 / 98$ à $22: 31$ & 100 & 71 & 71 & oui & $3 \mathrm{~m}^{3} / \mathrm{s}$ \\
\hline $15 / 04 / 98$ à $22: 46$ & 100 & 70 & 70 & oui & $3 \mathrm{~m}^{3} / \mathrm{s}$ \\
\hline $20 / 04 / 98$ à 21:36 & 100 & 63 & 63 & - & $3 \mathrm{~m}^{3} / \mathrm{s}$ \\
\hline $22 / 04 / 98$ à $22: 29$ & 100 & 66 & 66 & - & $3 \mathrm{~m}^{3} / \mathrm{s}$ \\
\hline 23/04/98 à 23:40 & 100 & 78 & 78 & - & $3 \mathrm{~m}^{3} / \mathrm{s}$ \\
\hline 28/0498 à 23:12 & 100 & 65 & 65 & - & entre 2 et $3 \mathrm{~m}^{3} / \mathrm{s}$ \\
\hline $29 / 04 / 98$ à $22: 24$ & 100 & 59 & 59 & - & entre 2 et $3 \mathrm{~m}^{3} / \mathrm{s}$ \\
\hline $04 / 05 / 98$ à $22: 45$ & 99 & 83 & 84 & - & $3 \mathrm{~m}^{3} / \mathrm{s}$ \\
\hline $06 / 05 / 98$ à $22: 28$ & 100 & 85 & 85 & - & $3 \mathrm{~m}^{3} / \mathrm{s}$ \\
\hline 11/05/98 à $21: 56$ & 98 & 81 & 81 & - & $3 \mathrm{~m}^{3} / \mathrm{s}$ \\
\hline Total (tous lots) & 1272 & 911 & 71,6 & & \\
\hline Total (sans pannes de vanne) & 797 & 580 & 72,8 & & \\
\hline
\end{tabular}



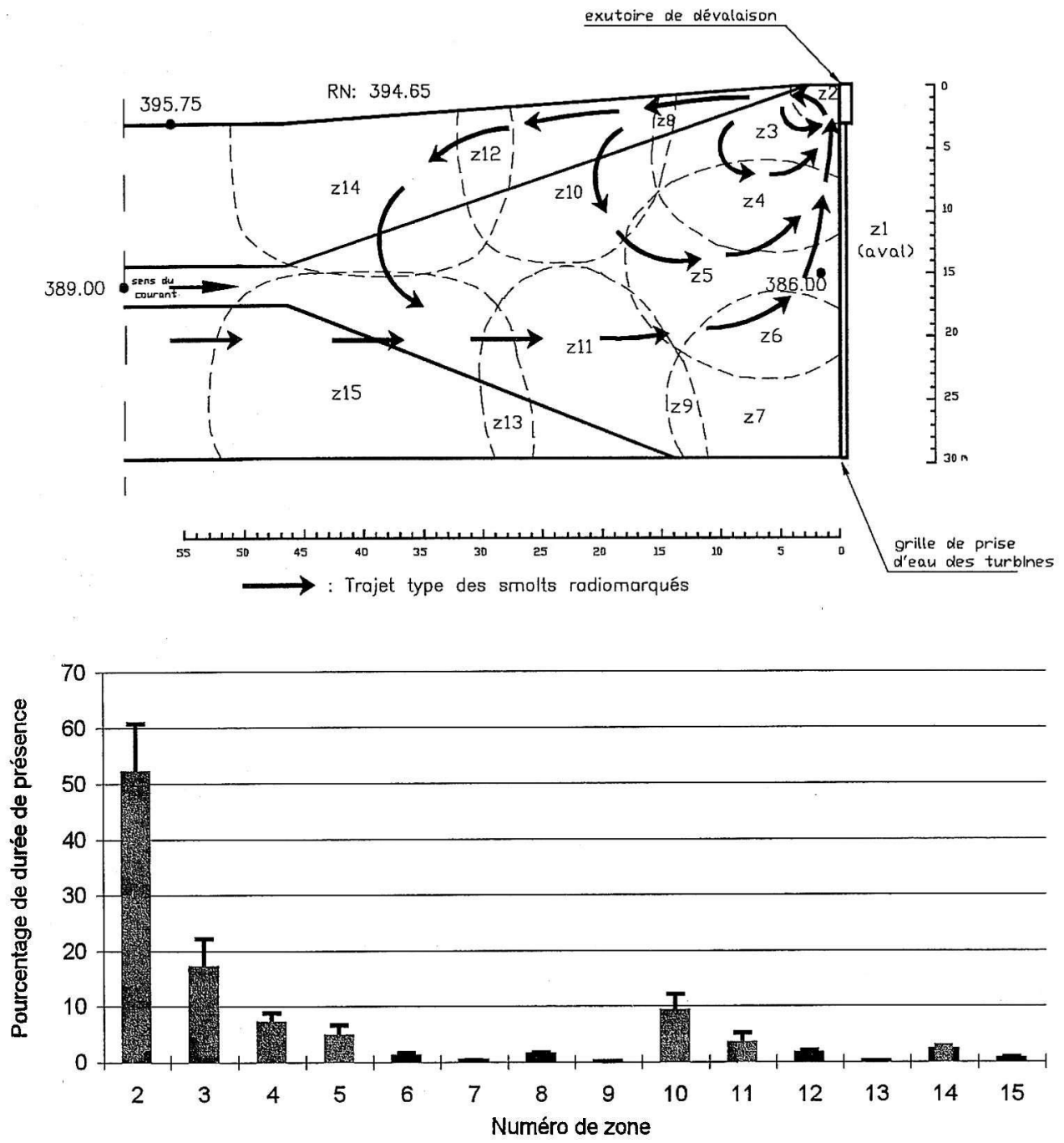

Figure 4

Zones de réception des radio-émetteurs, répartition des pourcentages de temps de présence des smolts radiomarqués dans ces différentes zones et trajet type.

\section{Figure 4}

Radiotelemetry listening areas, presence time percentages repartition of radiotagged smolts in these different areas and typical route.

\section{Efficacité de l'exutoire au cours des campagnes 1997 et 1998}

En 1997, sur les 1528 smolts lâchés, 1119 ont été récupérés dans le piège, ce qui correspond à une efficacité moyenne de l'exutoire de dévalaison de 73,2\% (Tableau II). Le pourcentage de recapture a varié de $57 \%$ à $84 \%$ selon les lots. Ce résultat a été obtenu alors que le débit de la Garonne était particulièrement faible (coefficients d'hydraulicité de 0,63 en avril et 0,61 en mai).

En 1998, sur les 1272 smolts lâchés, 911 ont emprunté l'exutoire. L'efficacité a oscillé entre $59 \%$ et $85 \%$, la moyenne étant de $71,6 \%$ (Tableau II). En retirant les résultats obtenus lors de lâchers perturbés par des pannes de la vanne de l'exutoire, l'efficacité du dispositif de dévalaison atteint une valeur moyenne de 72,8\% (580 smolts piégés sur 797 lâchés). Lors de cette campagne, les débits ont été représentatifs des conditions normales, les coefficients d'hydraulicité étant compris entre 0,94 et 1,00. 
Le débit turbiné à Camon a varié de $20 \mathrm{~m}^{3} / \mathrm{s}$ à $67 \mathrm{~m} 3 / \mathrm{s}$ au cours de la campagne 1997 et de $40 \mathrm{~m}^{3} / \mathrm{s}$ à $79 \mathrm{~m}^{3} / \mathrm{s}$ pour celle de 1998 . Le débit de l'exutoire étant de $3 \mathrm{~m}^{3} / \mathrm{s}$ pendant les 2 heures suivant le lâcher et limité à 2 m³/s le reste de temps en 1997 alors qu'il était fixé de manière constante à $3 \mathrm{~m}^{3 / \mathrm{s}}$ l'année suivante, ce sont respectivement $3 \%$ à $10 \%$ et $4 \%$ à $8 \%$ du débit turbiné à l'usine de Camon qui ont transité par l'exutoire pour chacune de ces deux études.

\section{Influence de la taille des poissons}

En 1996, l'influence de la taille des smolts a été étudiée sur 5 lots pour un total de 994 smolts. Parmi ces poissons, 370 ont été recapturés dans le piège associé à l'exutoire de dévalaison. La taille moyenne des smolts lâchés $(17,3 \mathrm{~cm})$ est inférieure à celle des individus piégés $(17,5 \mathrm{~cm})$. Cette différence, bien que faible, est cependant statistiquement significative (ANOVA, $F=5,43 ; p<0,05$ ).

L'analyse des fréquences par classes de taille des smolts lâchés et des smolts recapturés pendant la campagne 1996 (Figure 5) révèle que le pourcentage des poissons de taille inférieure à $16 \mathrm{~cm}$ est plus faible pour les individus recapturés que pour les smolts lâchés (WILCOXON, $Z=2,16 ; p<0,05$ ). Aucune différence sur les pourcentages de smolts appartenant aux classes de tailles supérieures à $16 \mathrm{~cm}$ n'a par contre été mise en évidence.

Les mêmes analyses, conduites sur les smolts lâchés et piégés pendant les campagnes 1997 et 1998, n'indiquent pas de différence de taille entre ces deux populations.

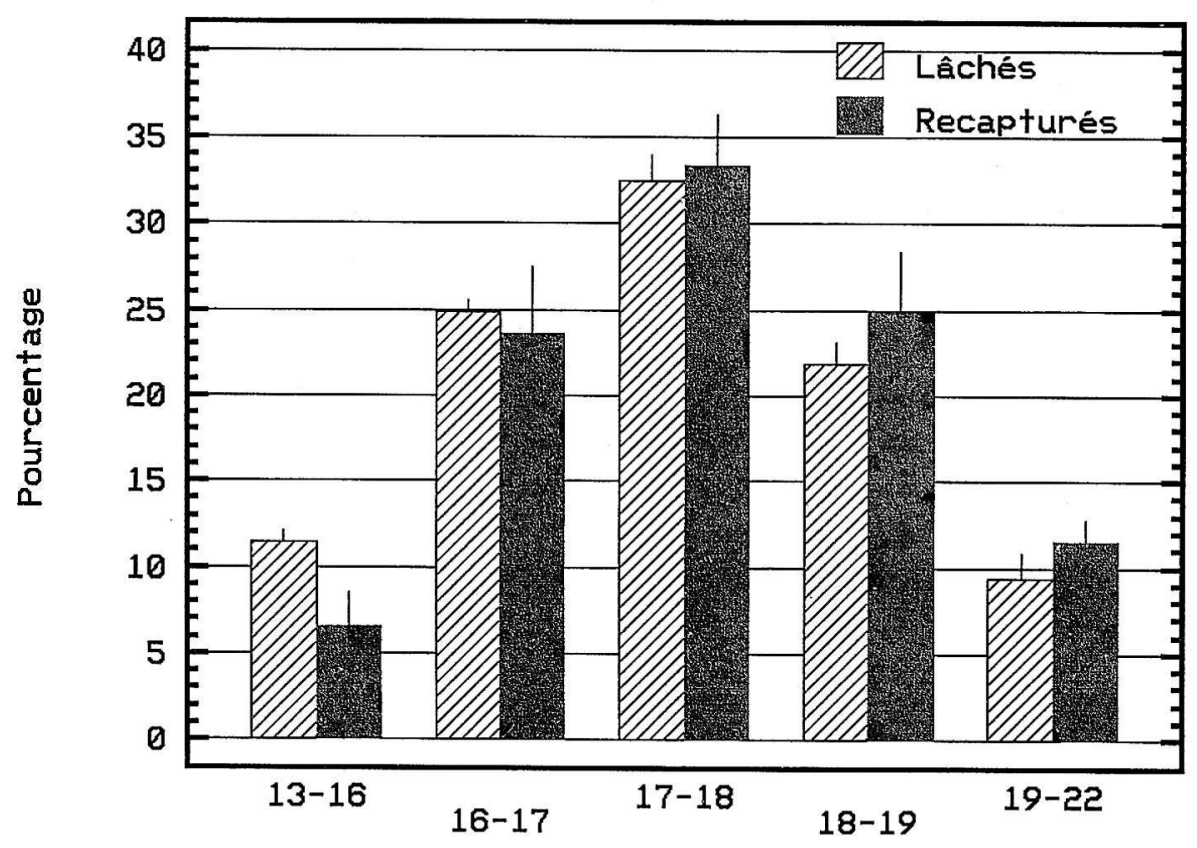

Figure 5

Répartition des tailles (longueur totale en $\mathrm{cm}$ ) des smolts lâchés et des smolts recapturés en 1996.

\section{Figure 5}

Length repartition (total length in $\mathrm{cm}$ ) of smolts released and captured in 1996. 


\section{Suivi de l'activité de dévalaison}

\section{Captures de poissons sauvages}

En plus des smolts de saumon atlantique lâchés pour les besoins de l'étude, 7715 salmonidés ont été capturés dans le piège associé à l'exutoire de Camon au cours des 3 campagnes. L'espèce présente en plus grand nombre était la truite commune (Salmo trutta L.) avec un total de 5433 captures. Quatre-vingt cinq pour cent de ces truites présentaient une robe plus ou moins argentée et brillante (stade truite blanchissante ou stade pré-smolt). Deux mille deux cent quatre-vingt deux smolts de saumon sauvages ont aussi été piégés.

\section{Activité quotidienne}

Le nombre maximum d'individus capturés en une journée peut représenter un pourcentage élevé de la totalité de la population dévalante. Ainsi, $18 \%$ des captures de la campagne 1998 et plus de $43 \%$ de celles de la campagne 1997 ont été réalisées en 24 heures. Ces pics de migration correspondent généralement à une augmentation du débit du cours d'eau de plusieurs dizaines de $\mathrm{m}^{3 / \mathrm{s}}$ et/ou une augmentation de la température (Figure 6).
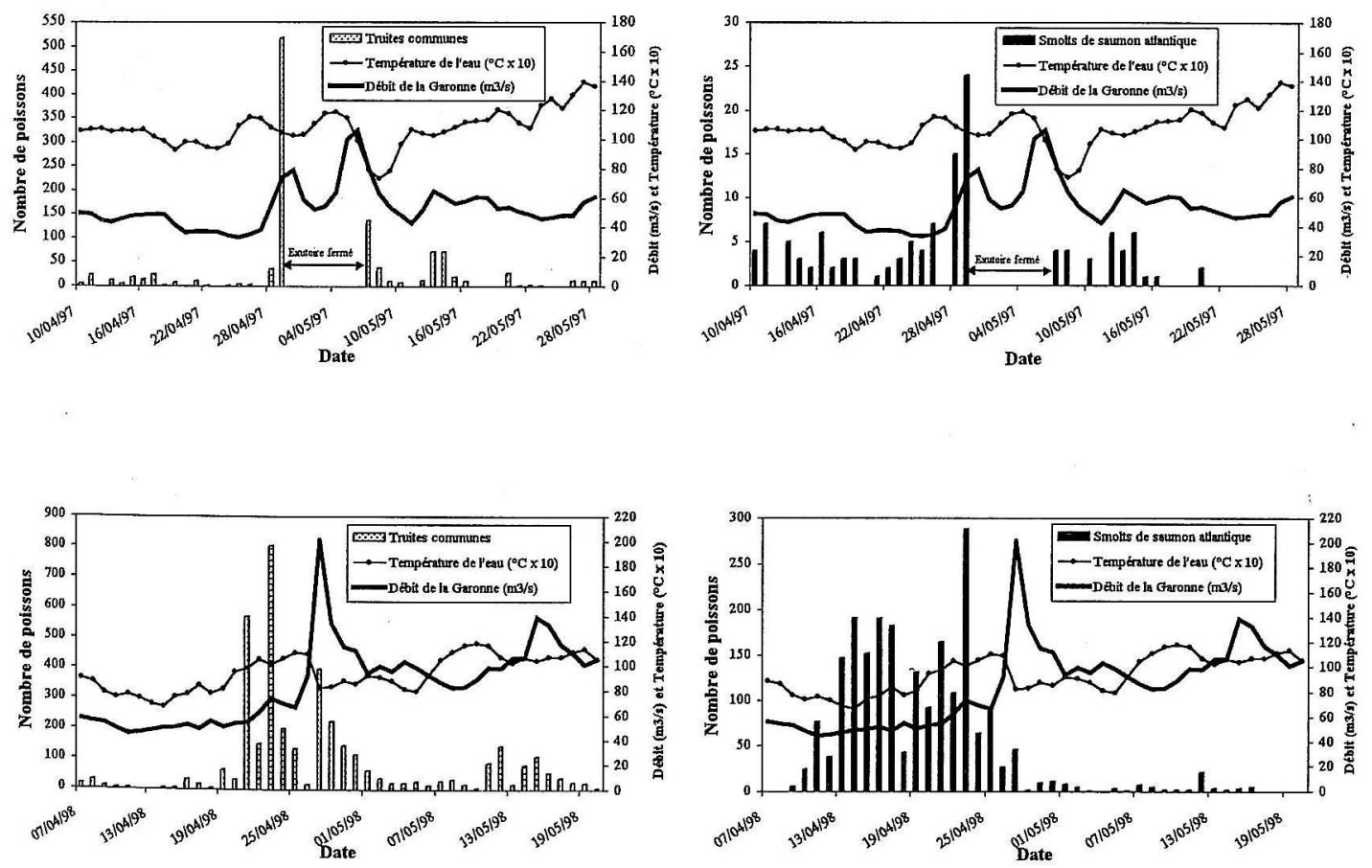

Figure 6

Captures quotidiennes de poissons sauvages lors des campagnes 1997 et 1998.

Figure 6

Daily wild fish catches during 1997 et 1998 studies. 


\section{Activité nycthémérale}

En 1998, l'enregistrement vidéo continu des passages dans l'exutoire a permis de connaitre l'horaire de dévalaison de 6642 poissons. La vidéo ne permettant toutefois pas de faire une distinction entre les différents salmonidés, cette observation concerne une population constituée à la fois de truites et de smolts de saumon atlantique. En nE considérant que les individus sauvages et les smolts lâchés ayant été recapturés les jours suivants leur remise à l'eau, il apparaît que $85 \%$ (soit 4804 individus) des poissons on: dévalé de nuit contre uniquement $15 \%$ (soit 846 individus) de jour.

\section{Influence de la lumière}

\section{Déplacements en amont de l'exutoire}

Bien que les zones préférentiellement fréquentées par les poissons équipés d'un émetteur soient celles situées à proximité de l'exutoire, les conditions d'éclairage ont toutefois influencé les pourcentages des durées de présence des smolts radiomarqués au niveau de ces différentes zones (Figure 7). Les smolts passent plus de temps dans la zone sous l'influence directe de l'éclairage (zone 2) quand la lampe fonctionne que quand elle est éteinte (WILCOXON, $Z=2,31 ; p<0,05)$.

L'activité nocturne des smolts dans les zones proches de la source lumineuse (zones 2, 3 et 4), quantifiée par le nombre de changements de zone par minute, est influencée par l'éclairage (WILCOXON, $Z=2,45 ; p<0,05$ ), les smolts radiomarqués effectuant en moyenne 3,6 changements de zone par minute lorsque la lampe est éteinte contre seulement 1,0 changement par minute quand elle fonctionne. Les graphes représentant les déplacements de smolts radiomarqués illustrent ce résultat (Figure 8 ). De nuit en l'absence d'éclairage, comme de jour, ces smolts ont tendance à se déplacer dans le canal d'amenée. Par contre, lorsqu'ils se présentent à proximité de la lampe alors que cette dernière fonctionne, ils restent principalement en zone 2 , dans le secteur directement éclairé.

Phase éclairée

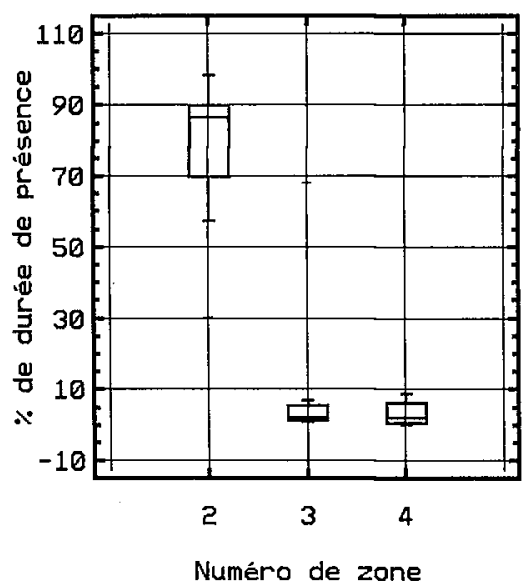

Phase éteinte

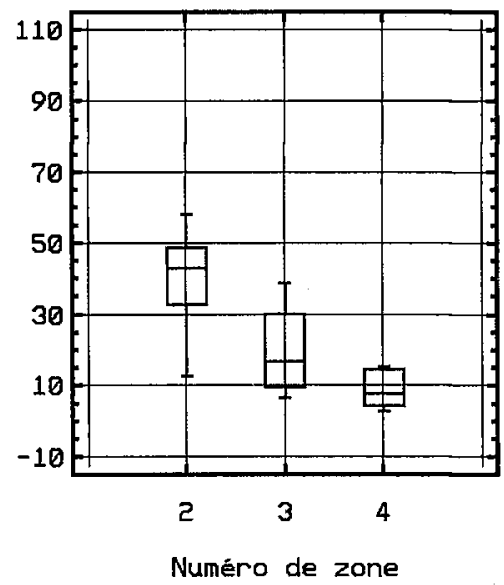

Figure 7

Comparaison des pourcentages de durée de présence des smolts radiomarqués au niveau des zones à proximité de l'exutoire en phase d'éclairage et d'extinction de la lampe à vapeur de mercure.

\section{Figure 7}

Comparison of presence time percentages of radiotagged smolts in areas located in the vicinity of the bypass entrance. 

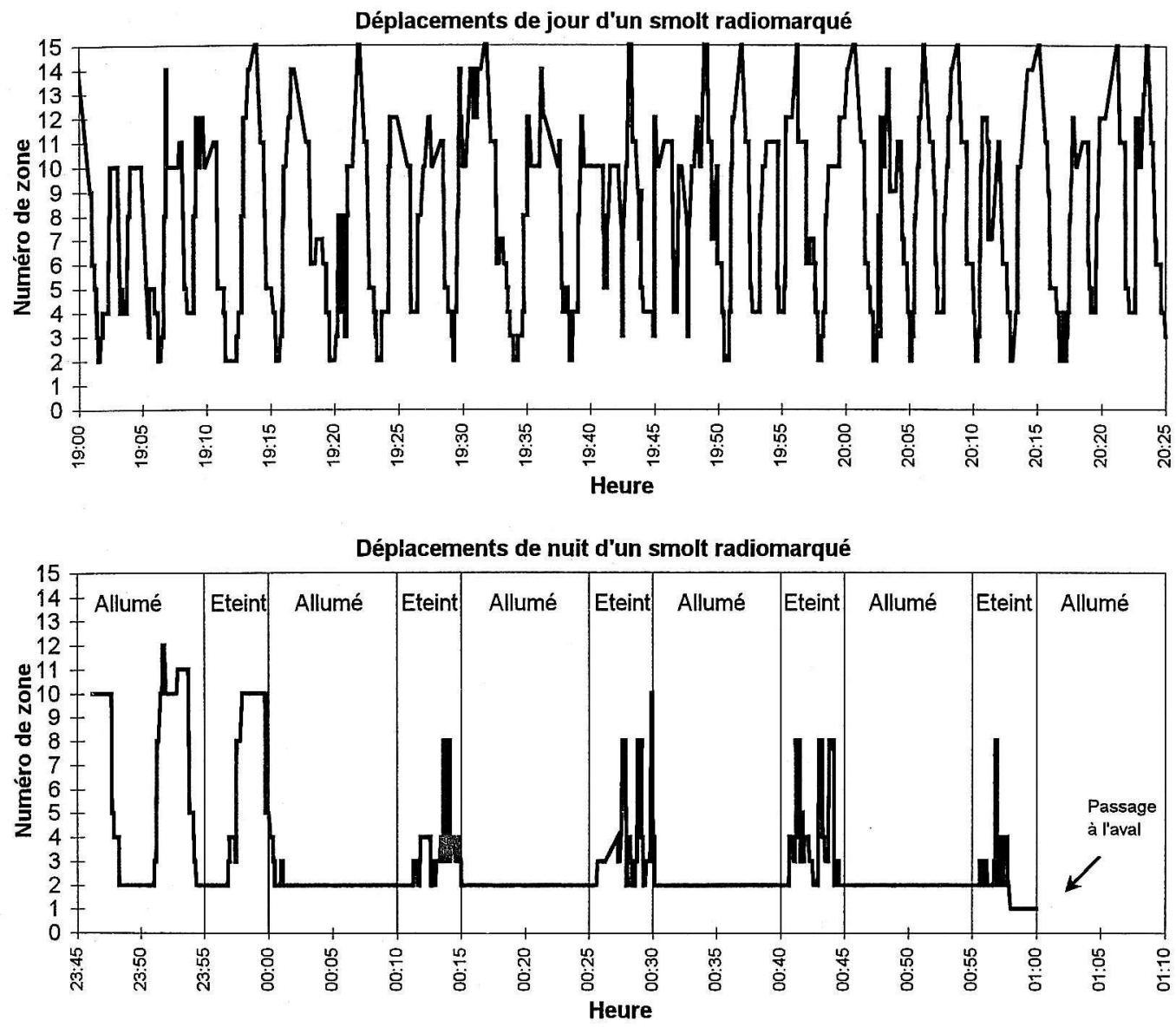

Figure 8

Exemple de graphe de déplacements d'un smolt radiomarqué de jour et de nuït avec un éclairage intermittent.

Figure 8

Diagram showing radiotagged smolt displacements during day and during night with intermittent lighting.

\section{Répartition des passages nocturnes}

Les enregistrements vidéo réalisés de nuit ont fourni une indication précise des horaires de passage des poissons dans le dispositif de dévalaison et donc des conditions d'éclairage lors de leur entrée dans l'exutoire. Cependant, ils n'ont pas permis de distinguer les saumons des truites. L'analyse suivante concerne donc les deux espèces.

Chacune des phases d'éclairage ou d'extinction des campagnes 1996 et 1997 peut être divisée en périodes plus courtes de 2 min $30 \mathrm{~s}$ codées $\mathrm{A} 1, \mathrm{~A} 2, \mathrm{~A} 3, \mathrm{~A} 4$ pour la phase pendant laquelle la lampe est allumée et E1, E2 pour la phase pendant laquelle elle est éteinte. Il apparaît une différence significative des passages de poissons dans l'exutoire en fonction des conditions lumineuses, que la lampe soit installée en amont de l'entrée du dispositif de dévalaison (ANOVA, $F=12,81 ; p<0,001$ ) ou en aval de cette entrée (ANOVA, $F=25,60 ; p<0,001$ ). Les poissons pénètrent dans l'exutoire principalement lors de la condition E1, c'est-à-dire immédiatement après l'extinction de la lampe (Figure 9). 
En 1998, les 2 lampes ont été utilisées. Après un éclairage simultané de ces 2 lampes (condition L1 + L2), seule celle placée la plus en aval a été maintenue éclairée (condition L2) avant de s'éteindre à son tour (condition E). Dans ces conditions, le nombre de poissons pénétrant dans le dispositif est significativement plus important après l'extinction de la lampe L1 (ANOVA, $F=58,11 ; p<0,001$ ). En moyenne, 0,21 passage par minute a été observé lors de l'éclairage simultané des 2 lampes et 0,51 passage par minute après l'arrêt de la première (Figure 10). A l'extinction de la deuxième lampe, les passages ont été significativement plus nombreux que lorsqu'elle était seule en fonctionnement (ANOVA, $F=131,53 ; p<0,001$ ) : en moyenne, 1,3 passage par minute a alors été comptabilisé. Le maximum de passages est donc intervenu lors de l'obscurité totale.

En 1998, sur des périodes où la population dévalante était constituée à plus de $80 \%$ de smolts, le nombre de passages par minute a augmenté après l'extinction de la première lampe (ANOVA, $F=30,44 ; p<0,001$ ) ainsi qu'après celle de la seconde (ANOVA, $F=53,87 ; p<0,001$ ). Le même phénomène a été observé sur une population constituée à plus de $70 \%$ de truites blanchissantes.
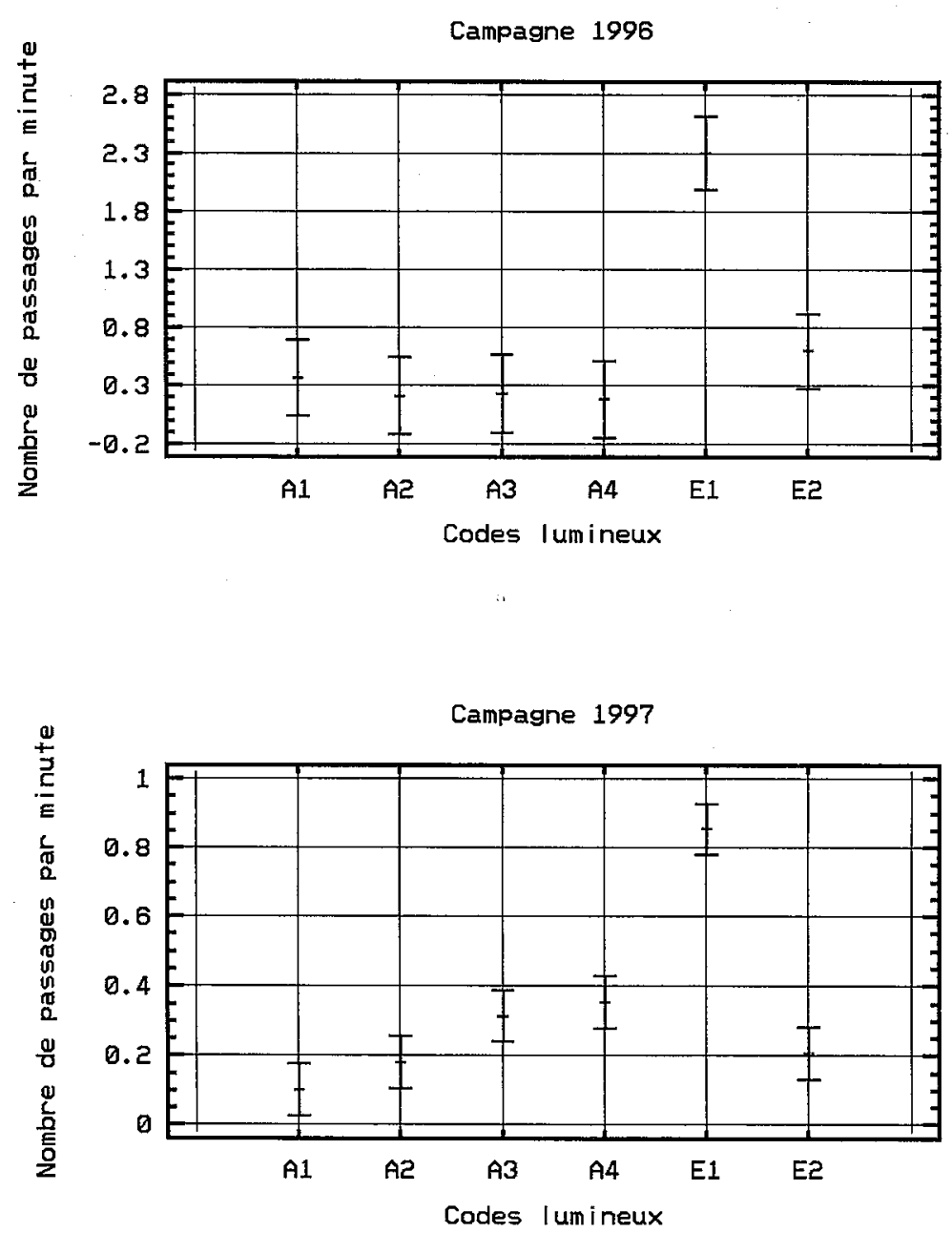

\section{Figure 9}

Nombre de poissons piégés par minute en fonction des conditions lumineuses au niveau de l'entrée de l'exutoire en 1996 et en 1997.

\section{Figure 9}

Number of fish captured per minute according to light conditions at the bypass entrance in 1996 and 1997. 


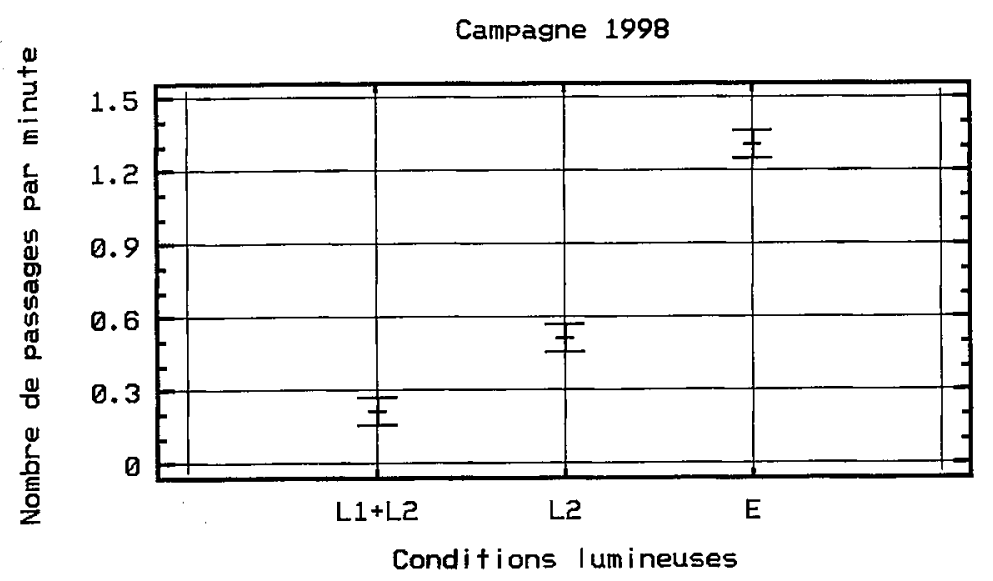

Figure 10

Nombre de poissons piégés par minute en fonction des conditions lumineuses au niveau de l'entrée de l'exutoire en 1998.

\section{Figure 10}

Number of fish captured per minute according to the light conditions at the bypass entrance in 1998.

\section{DISCUSSION}

Une première évaluation de l'exutoire de dévalaison installé au niveau de la prise d'eau de l'usine hydroélectrique de Camon a été effectuée au printemps 1996 ; les opérations de marquage-recapture ont mis en évidence une efficacité insuffisante avec une valeur moyenne de $34 \%$. Le suivi des déplacements de smolts à l'aide de la technique de radio-pistage dans une zone d'une cinquantaine de mètres en amont des grilles de la prise d'eau a montré que l'implantation de l'exutoire était correcte, les conditions hydrauliques dans le canal d'amenée guidant les poissons à proximité du dispositif de dévalaison. Bien que le débit prélevé alors par l'exutoire représente $2 \%$ à $4 \%$ du débit turbiné et soit compris dans la plage de valeurs communément admise pour un dispositif de dévalaison (FERGUSON, POE et CARLSON, 1996 ; LARINIER et TRAVADE, 1997), un tel pourcentage s'est avéré insuffisant pour permettre une bonne efficacité de l'exutoire. Une ascendance réduit la zone d'influence de l'exutoire et peut expliquer les difficultés rencontrées par les poissons à s'engager dans le dispositif.

La mise en place d'une grille de tranquillisation et de platelages horizontaux en amont immédiat de l'exutoire, associée à une augmentation du débit de ce dernier ( $3 \%$ à $10 \%$ du débit turbiné), a permis d'étendre la zone d'influence du dispositif de dévalaison. Cette modification s'est traduite par une augmentation significative de l'efficacité du dispositif qui a atteint la valeur moyenne de $73 \%$, aussi bien lorsque les débits turbinés étaient faibles (campagne 1997) qu'élevés (campagne 1998).

Un effet de la taille des smolts lâchés sur l'efficacité de l'exutoire de dévalaison a pu être mis en évidence en 1996. Les individus de taille inférieure à $16 \mathrm{~cm}$ ont été récupérés en moins grande proportion dans le piège que les poissons de taille supérieure. Les grilles de protection de la prise d'eau de l'usine, d'un espacement entre barreaux de $4 \mathrm{~cm}$, semblent exercer un effet répulsif moins prononcé sur ces individus de petite taille. Des constatations identiques ont été réalisées au niveau d'autres prises d'eau (CHANSEAU, LARINIER et TRAVADE, 1999). 
En plus des smolts lâchés pour les études, 7715 salmonidés ont emprunté le dispositif de dévalaison de Camon. Lors des 3 années d'étude, la majorité de cette population était composée de truites communes blanchissantes. En 1998, un effectif non négligeable de smolts sauvages a aussi été observé. La quasi-totalité (94\%) de ces: smolts provenait de déversements de poissons âgés d'un an, réalisés en avril 1998 à une: dizaine de kilomètres en amont de la zone d'étude. La dévalaison des truites blanchissantes semble se réaliser en même temps que celle des smolts de saumor: atlantique, comme l'ont déjà noté BERG et JONSSON (1989).

La majorité des passages (85\%) s'est réalisée de nuit. Ce résultat est concordan avec les observations de divers auteurs qui ont mentionné que la dévalaison étai principalement nocturne pour les smolts de saumon que ce soit en laboratoire (THORPE. 1989) ou en milieu naturel (THORPE et al., 1981 ; YOUNGSON et al., 1983 HESTHAGEN et GARNÅS, 1986 ; BAKSHTANSKY, NESTEROV et NEKLYUDOV, 1987) ainsi que pour les truites blanchissantes (EUZENAT et FOURNEL, 1976), les smolts de ces deux espèces présentant des rythmes circadiens quasi identiques (LEONKO et CHERNITSKY, 1986).

L'utilisation nocturne d'un éclairage intermittent de l'entrée de l'exutoire a eu les mêmes effets que ceux observés par CHANSEAU, LARINIER et TRAVADE (1999) sur l'activité des smolts radiomarqués dans les zones directement soumises aux variations des conditions lumineuses et sur le rythme de passage des poissons dans le dispositif de dévalaison. Sur le site de Camon, le fonctionnement de la lampe a eu aussi un effet sur le stationnement des poissons dans les différentes zones de réception, ces derniers fréquentant plus longuement le secteur soumis aux variations d'éclairage quand la lampe était allumée.

Lorsque deux lampes ont été employées (campagne 1998), l'extinction anticipée de celle située la plus en amont était certes suivie d'une augmentation du nombre de passages à l'exutoire, mais cette augmentation restait toutefois moins prononcée que celle observée lors de l'extinction de la seconde lampe installée plus en aval, les poissons étant alors plongés dans l'obscurité totale. La présence de cette seconde lampe limitait les passages par l'exutoire, les poissons semblant alors se concentrer au-dessous de cette dernière. Il pourrait alors être envisagé d'assurer le guidage de smolts par l'intermédiaire d'une rampe de lampes à vapeur de mercure de faible puissance dont chacune s'éteindrait de manière successive. Un tel dispositif pourrait présenter une certaine efficacité au niveau de sites où les vitesses d'écoulement restent limitées.

Bien que l'utilisation de lampes à vapeur de mercure modifie le comportement et le rythme de passage des poissons dans le dispositif de dévalaison, il reste toutefois à démontrer une éventuelle amélioration systématique de l'efficacité d'un exutoire par la mise en place d'un éclairage intermittent au niveau de son entrée. Certains travaux récents indiquent que la lumière est susceptible d'améliorer l'efficacité d'un dispositif de dévalaison lorsque les vitesses de l'écoulement sont limitées (GOSSET et al., 1997) alors qu'aucune amélioration n'est décelable avec des vitesses plus prononcées (GOSSET et al., 1998). Ces travaux sont à poursuivre et permettront d'identifier l'intérêt et les limites d'un tel éclairage.

\section{CONCLUSION}

Les trois années d'expérimentation ont montré qu'un seul exutoire pouvait permettre la dévalaison de manière efficace de la grande majorité des smolts au niveau d'une prise d'eau d'une trentaine de mètres de largeur, beaucoup plus large que celles qui avaient faiì l'objet de tests jusqu'alors en France. Cette efficacité est cependant liée à l'existence dans 
le canal d'amenée de conditions hydrauliques favorables, concentrant les poissons à proximité de l'entrée de l'exutoire (LARINIER et TRAVADE, 1997 ; CHANSEAU, LARINIER et TRAVADE, 1999). Ces conditions hydrodynamiques, que l'on pourrait qualifier de "lointaines », ne sont cependant pas suffisantes et doivent être impérativement associées à des conditions hydrauliques favorables à proximité immédiate de l'entrée de l'exutoire, la zone d'influence de celui-ci, c'est-à-dire la zone dans laquelle le poisson est susceptible de percevoir l'entrée de l'exutoire, devant s'étendre jusqu'à la zone de concentration des poissons.

\section{REMERCIEMENTS}

Ces études ont bénéficié de l'aide technique de S. BOSC, L. CARRY, E. GALIAY, et G. OULES.

Nous remercions MI.GA.DO. pour avoir assuré la maîtrise d'ouvrage de l'étude, la FDPPMA de la Haute-Garonne pour avoir mis à notre disposition des bassins de la pisciculture de Soueich ainsi que EDF, en particulier le GEH de Saint-Gaudens et l'ensemble du personnel de l'usine de Camon.

\section{BIBLIOGRAPHIE}

BAKSHTANSKY E.L., NESTEROV V.D., NEKLYUDOV M.N., 1987. Development of schooling behavior in juvenile Atlantic salmon, Salmo salar, during seaward migration. Vopr. ikhtiologii, 6, 1000-1009.

BERG O.K., JONSSON B., 1989. Migratory patterns of anadromous Atlantic salmon, brown trout and Arctic charr from the Vardnes River in northern Norway. In : BRANNON E., JONSSON B. (Eds.), Proceedings of the salmonid migration and distribution symposium. June 23-25, 1987. Second international symposium, 106-115, School of Fisheries, University of Washington, Seattle.

CHANSEAU M., LARINIER M., TRAVADE F., 1999. Efficacité d'un exutoire de dévalaison pour smolts de saumon atlantique (Salmo salar L.) et comportement des poissons au niveau de l'aménagement hydroélectrique de Bedous sur le Gave d'Aspe étudiés par la technique de marquage-recapture et par radiotélémétrie. Bull. Fr. Pêche Piscic., 353/354, 99-120.

EPRI, 1994. Research update on fish protection technologies for water intakes. EPRI, TR-104122, $208 \mathrm{p}$.

EUZENAT G., FOURNEL F., 1976. Recherches sur la Truite commune (Salmo trutta, L.) dans une rivière de Bretagne, le Scorff. Thèse Doct. 3e cycle, U.E.R. des Sciences du Comportement et de l'Environnement, Univ. Rennes, 243 p.

FERGUSON J.W., POE T.P., CARLSON T.J., 1996. Surface-oriented bypass systems for juvenile salmonids on the Colombia River, USA. In : JUNGWIRTH M., SCHMUTZ S., WEISS S. (Eds.), Fish migration and fish bypasses, Fishing News Books, 281-299.

GOSSET C., TRAVADE F., VOISIN M., GARAICOECHEA C., 1997. Etude de dispositifs de dévalaison pour smolts de saumon atlantique. Centrale hydroélectrique de Halsou (Nive-64). INRA, Station d'hydrobiologie de Saint-Pée-sur-Nivelle, $42 \mathrm{p}$.

GOSSET C., TRAVADE F., LABASTIE C., GARAICOECHEA C., 1998. Etude de dispositifs de dévalaison pour smolts de saumon atlantique : influence de la lumière sur l'efficacité des exutoires de dévalaison. Centrale hydroélectrique de Halsou (Nive-64). INRA, Station d'hydrobiologie de Saint-Pée-sur-Nivelle, 34 p. 
HESTHAGEN T., GARNÅS E., 1986. Migration of Atlantic salmon smolts in River Orkla o: central Norway in relation to management of a hydroelectric station. N. Am. J. Fist. Manage., 6, 376-382.

LARINIER M., BOYER-BERNARD S., 1991a. Dévalaison des smolts et efficacité d'ur. exutoire de dévalaison à l'usine hydroélectrique d'Halsou sur la Nive. Bull. $\mathrm{Fl}$ Pêche Piscic., 321, 72-92.

LARINIER M., BOYER-BERNARD S., 1991b. Dévalaison des smolts de saumon atlantique au barrage de Poutès sur l'Allier (43) : utilisation de lampes à vapeur de mercure er: vue d'optimiser l'efficacité de l'exutoire de dévalaison. Bull. Fr. Pêche Piscic., 323 129-148.

LARINIER M., TRAVADE F., 1997. The development and evaluation of downstrear bypasses for juvenile salmonids at small hydroelectric plants in France. Americar: Fisheries Society, 127th Annual Meeting - Monterey, California, August 24-28. 1997, $18 \mathrm{p}$.

LEONKO A.A., CHERNITSKY A.G., 1986. Comparative analysis of smolt migration of Atlantic salmon, Salmo salar, and Sea Trout, Salmo trutta. Vopr. ikhtiologii, 5 , 6-113.

TAFT N., 1988. Evaluations of fish protection systems for use at hydroelectric plants. Hydro Review, 54-62.

THORPE J.E., 1989. Downstream migration of young salmon : recent findings, with special reference to Atlantic salmon, Salmo salar L. In : BRANNON E., JONSSON B. (Eds.), Proceedings of the salmonid migration and distribution symposium. June 23-25, 1987. Second international symposium, 81-86, School of Fisheries, University of Washington, Seattle.

THORPE J.E., ROSS L.G., STRUTHERS G., WATTS W., 1981. Tracking Atlantic salmon smolts, Salmo salar L., through Loch Voil, Scotland. J. Fish Biol., 19, 519-537.

TRAVADE F., LARINIER M., INGENDAHL D., BACH J.M., PUJO D., BARRACOU D., 1996. Expérimentation d'un dispositif de dévalaison pour les juvéniles de saumon atlantique (année 1994). Usine hydroélectrique de Soeix (Gave d'Aspe-64). Rapport GHAAPPE 96.02, 34 p.

YOUNGSON A.F., BUCK R.J.G., SIMPSON T.H., HAY D.W., 1983. The autumn and spring emigrations of juvenile Atlantic salmon, Salmo salar L., from the Girnock Burn, Aberdeenshire, Scotland : environmental release of migration. J. Fish Biol., 23, 625-639. 